\title{
Type-3 snakes in high energy accelerators
}

\author{
Vladimir A. Anferov* \\ Physics Department, University of Michigan, Ann Arbor, Michigan 48109-1120
}

(Received 20 July 1998; published 16 November 1998)

\begin{abstract}
Several pairs of Siberian snakes are usually required to preserve the beam polarization in a high energy proton ring. It is found that installing additional type-3 snakes in the arcs of a high energy accelerator could significantly reduce local spin perturbation between snakes and thus improve overall spin stability in the ring. The best results could be obtained when a type- 3 snake is installed $1 / 4$ of the way into each arc. [S1098-4402(98)00021-4]
\end{abstract}

PACS numbers: 41.75.Ak, 29.27.Bd, 29.27.Hj

\section{INTRODUCTION}

Accelerating polarized proton beams to high energies requires overcoming many spin-depolarizing resonances. The Siberian snakes method [1] allows one to reorganize the spin motion to avoid crossing the depolarizing resonances during the acceleration [2]. Each snake rotates the proton's spin by $180^{\circ}$ while keeping the beam orbit unperturbed outside the snake. Conventional Siberian snakes have their axes of spin rotation in the horizontal plane of a circular accelerator. Historically, a snake with the axis of spin rotation along the beam direction was called a type1 snake, while a snake with the radial axis was defined as type-2. A pair of type-1 and type- 2 snakes installed in the opposite straight sections of a ring would make the amount of spin precession in each turn independent of energy and would cancel the depolarization effect over two turns around the ring. However, the strength of the spin perturbation increases with energy. While two snakes can preserve the beam polarization up to about $200 \mathrm{GeV}$ (as in RHIC [3] at Brookhaven), at higher energies the spin perturbation within one turn could be large enough to cause depolarization.

To reduce the amount of spin perturbation between two snakes, one can increase the number of snakes in the ring. In this paper we consider another possibility of improving the spin stability in a ring with Siberian snakes by installing additional type-3 snakes [4] with vertical axis of spin rotation. While type-3 snakes cannot make the spin tune energy independent and do not prevent crossing of the depolarizing resonances, they could significantly reduce the amount of spin perturbation at each depolarizing resonance.

\footnotetext{
*Telephone: (734) 764-5114. Fax: (734) 763-9027.

Email address: anferov@umich.edu
}

\section{SPIN PERTURBATION IN A PERIODIC ACCELERATOR LATTICE}

In a ring without Siberian snakes, an unperturbed spin precesses around the vertical dipole field. However, even in an ideal ring without magnet errors, this vertical spin precession can be perturbed by periodic horizontal fields that protons encounter in the ring's quadrupoles. The strength of the spin perturbation in a ring is given by the spin-orbit coupling integral [5]

$$
I^{ \pm}=\int e^{i \psi(s)} g(s) \sqrt{\beta_{z}(s)} e^{ \pm i \psi_{z}(s)} d s,
$$

where $\psi$ is the integrated phase of the spin precession, $g$ is the quadrupole strength, and $\beta_{z}$ and $\psi_{z}$ are the beta function and the integrated phase for the vertical betatron oscillations. Over $N$ identical FODO cells, the spin-orbit coupling integral is proportional to the spin perturbation in one cell

$$
\begin{aligned}
I^{ \pm} & =\sum_{k=0}^{N-1} e^{k 2 \pi i\left(\nu_{1} \pm \mu_{z}\right)} \int_{\text {cell }} g(s) \sqrt{\beta_{z}} e^{i\left(\psi \pm \psi_{z}\right)} d s \\
& =\frac{1-e^{N 2 \pi i\left(\nu_{1} \pm \mu_{z}\right)}}{1-e^{2 \pi i\left(\nu_{1} \pm \mu_{z}\right)}} I_{\text {cell }}^{ \pm},
\end{aligned}
$$

where $\mu_{z}$ and $\nu_{1}$ are the betatron and spin phase advances in each cell. The fractional factor in Eq. (2) describes the resonant structure of the spin perturbation, which reaches maximum when the spin motion is synchronized with the betatron motion

$$
\nu_{1} \pm \mu_{z}=m
$$

where $m$ is an integer. Near such spin-orbit resonance, the spin perturbations from all cells add up coherently, and the effect of one cell is enhanced by a factor $\xi$,

$$
\begin{aligned}
\left.\xi(N)\right|_{\nu_{1} \pm \mu_{z}=m} & =\left|\frac{1-e^{N 2 \pi i\left(\nu_{1} \pm \mu_{z}\right)}}{1-e^{2 \pi i\left(\nu_{1} \pm \mu_{z}\right)}}\right| \\
& =\frac{\sin \left[N \pi\left(\nu_{1} \pm \mu_{z}\right)\right]}{\sin \left[\pi\left(\nu_{1} \pm \mu_{z}\right)\right]}\left|e^{i \pi(N-1)\left(\nu_{1} \pm \mu_{z}\right)}\right| \\
& =N .
\end{aligned}
$$


The regions of strong spin perturbations given by Eq. (3) are called intrinsic spin depolarizing resonances. Misalignments and field errors in the ring's magnets create closed orbit errors and cause imperfection depolarizing resonances. It is known that the strongest harmonic for the closed orbit errors is located at an integer nearest to the betatron tune. Thus, strong intrinsic and strong imperfection depolarizing resonances create spin perturbation at close frequencies, and the same treatment should work for the resonances of both types. A large number of the depolarizing resonances is crossed during acceleration due to the energy dependent rate of the vertical spin precession. The spin precession phase advance in a cell can be written as

$$
\nu_{1}=G \gamma \beta_{1},
$$

where $\beta_{1}$ is the orbit bending angle of the cell, $G=1.793$ is the proton's anomalous magnetic moment, and $\gamma$ is the Lorentz energy factor. Away from the resonances given by Eq. (3), the depolarization enhancement factor $\xi$ has $N-1$ minima with $\xi$ reaching zero at $\nu_{1} \pm$ $\mu_{z}=k / N(k=1,2, \ldots, N-1)$. Steffen noted [6] that these minima determine special energies, where the spin perturbation vanishes over $N$ cells, and, therefore, a long lifetime of the beam polarization could be obtained. One could say that the spin motion at these energies is "matched" with the betatron motion to provide maximum spin stability. In this paper we consider how a type-3 snake can improve local spin "matching" to increase spin stability in accelerators with Siberian snakes.

\section{TYPE-3 SNAKE EFFECT ON SPIN PERTURBATION}

The strongest spin perturbation occurs near the spinorbit resonances. Therefore, to improve spin stability in a ring with Siberian snakes one needs a method of local correction of the spin-orbit resonances. A type-3 snake can significantly reduce the spin perturbation near the strong depolarizing resonances given by Eq. (3); thus, installing a type- 3 snake between two regular Siberian snakes could improve overall spin stability. When a type- 3 snake is installed at the center of an arc consisting of $N=2 n$ FODO cells, the spin-orbit coupling integral becomes

$$
I^{ \pm}=\left(1+e^{i\left[\phi_{3}+2 \pi n\left(\nu_{1} \pm \mu_{z}\right)\right]}\right) I_{n}^{ \pm} \text {cells },
$$

where $\phi_{3}$ is the angle of spin rotation in the type- 3 snake and $I_{n}^{ \pm}$cells is the spin-orbit integral over $1 / 2$ of the arc. Equation (6) indicates that a full type-3 snake $\left(\phi_{3}=\pi\right)$ completely cancels the main spin-orbit resonances given by Eq. (3). However, the neighboring depolarizing resonances would be enhanced. For instance, the resonance

$$
\nu_{1} \pm \mu_{z}=m+\frac{1}{N}
$$

will get stronger in the presence of a full type-3 snake. To minimize local spin perturbation in the presence of a type-3 snake, we study how the depolarization enhancement factor $\xi$ changes when both the strength of a type-3 snake and its position in the arc are varied. As an example, we consider a regular accelerator arc consisting of 24 FODO cells with each cell providing a $90^{\circ}$ betatron phase advance.

When a type- 3 snake's spin rotation is $\pi-\delta$, the main spin-orbit resonances are no longer canceled, and the spin perturbation increases in the region

$$
\nu_{1} \pm \mu_{z}=m-\frac{1-\frac{\delta}{\pi}}{N} .
$$

This effect is illustrated in Fig. 1, which compares the depolarization enhancement factors for the cases of a full and a $120^{\circ}$ type- 3 snake installed at the center of the 24 FODO cells arc. The maximum spin perturbation still occurs near integer values of $\left(\nu_{1} \pm \mu_{z}\right)$; however, it is significantly smaller with a full type-3 snake in the arc than with a partial type-3 snake. Note that the depolarization enhancement factor depends only on relative phases of the spin and betatron motion and provides an energy independent way to analyze spin perturbation. Thus, only the fractional part of $\left(\nu_{1} \pm \mu_{z}\right)$ is important in the enhancement factor analysis, and the abscissa in Fig. 1 could be shifted by an arbitrary integer number.

The position of a type- 3 snake in the arc could be varied in steps of a full FODO cell or by one quadrupole (half a cell). However, if a type-3 snake is displaced by one quadrupole, then the spin-orbit coupling integral cannot be minimized simultaneously for $\nu_{1}+\mu_{z}=m$ and $\nu_{1}-\mu_{z}=m$ resonances

$$
\begin{aligned}
I^{ \pm}= & \left(1+e^{i\left[\phi_{3} \pm 2 \pi \mu_{z}+2 \pi n\left(\nu_{1} \pm \mu_{z}\right)\right]}\right) I_{\frac{N}{2}}^{ \pm} \text {cells } \\
& +\left(1-e^{i \phi_{3}}\right) g_{1} \sqrt{\beta_{1}},
\end{aligned}
$$

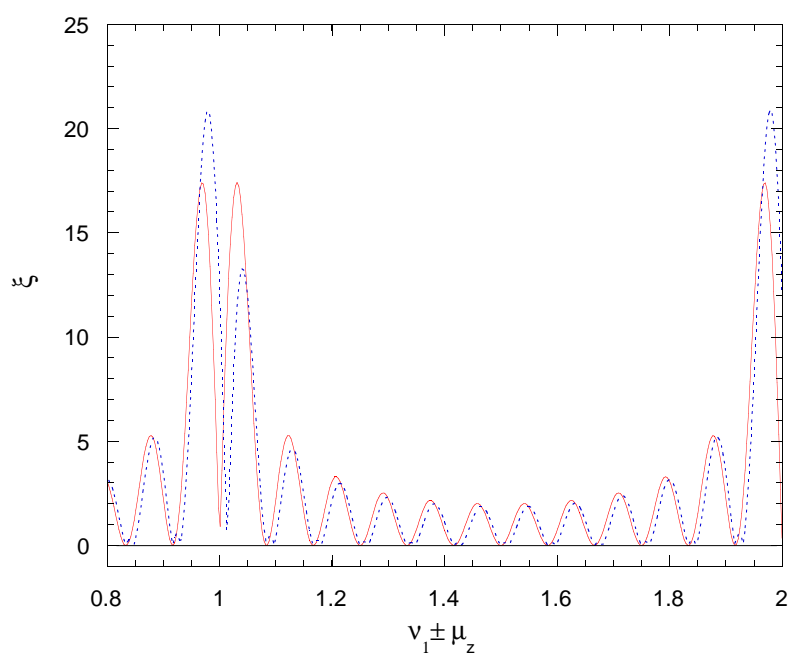

FIG. 1. (Color) The spin perturbation enhancement factor is plotted against $\nu_{1} \pm \mu_{z}$, when a type-3 snake is installed at the center of a 24 FODO cells arc. The solid curve corresponds to a full type- 3 snake. The dashed curve corresponds to a $120^{\circ}$ type-3 snake. 
where $g_{1}$ and $\beta_{1}$ are the strength and the vertical beta function in the offset quadrupole. To have depolarizing resonances with both signs of $\mu_{z}$ equally reduced, we will vary the location of a type- 3 snake by an integer number of cells.

When a full type- 3 snake is installed after the $n$th cell, the spin-orbit coupling integral can be written as

$$
\begin{aligned}
I^{ \pm} & =\left(1+e^{i\left[\pi+2 \pi n\left(\nu_{1} \pm \mu_{z}\right)\right]}\right) I_{n \text { cells }}^{ \pm}+I_{(24-2 n) \text { cells }}^{ \pm} \\
& =\xi_{n}\left(\nu_{1} \pm \mu_{z}\right) I_{\text {cell }}^{ \pm} .
\end{aligned}
$$

For each location of a full type-3 snake $(n=0,1, \ldots, 12)$, the maximum value of the enhancement factor $\xi_{n}\left(\nu_{1} \pm\right.$ $\left.\mu_{z}\right)$ was calculated in the region $0.8 \leq\left(\nu_{1} \pm \mu_{z}\right) \leq 2$. The results are plotted against the location of a full type3 snake in Fig. 2. The minimum spin perturbation is achieved when a full type- 3 snake is installed after six cells, which is $1 / 4$ of the arc. In this case, a type- 3 snake cancels the effect of the main spin-orbit resonances in the first half of the arc, while in the second half only the main spin-orbit resonances contribute to spin perturbation. In this optimal configuration, the type- 3 snake reduces the maximum spin perturbation in the arc by a factor of 2 .

We have considered using a single type- 3 snake in each arc to suppress the spin perturbation effects. Similarly, one can also install several type-3 snakes in each arc. In the extreme, a type- 3 snake could be installed in the middle of each quadrupole as proposed in [7]; then, the spin perturbation could be reduced by a factor of 50 . The required number of type- 3 snakes is determined by the following spin stability criteria.

When the betatron tunes are set far from the highorder snake resonances [8], the spin stability in a ring with Siberian snakes is determined by the amount of spin perturbation between the snakes. The angle of spin perturbation between the snakes should always be below

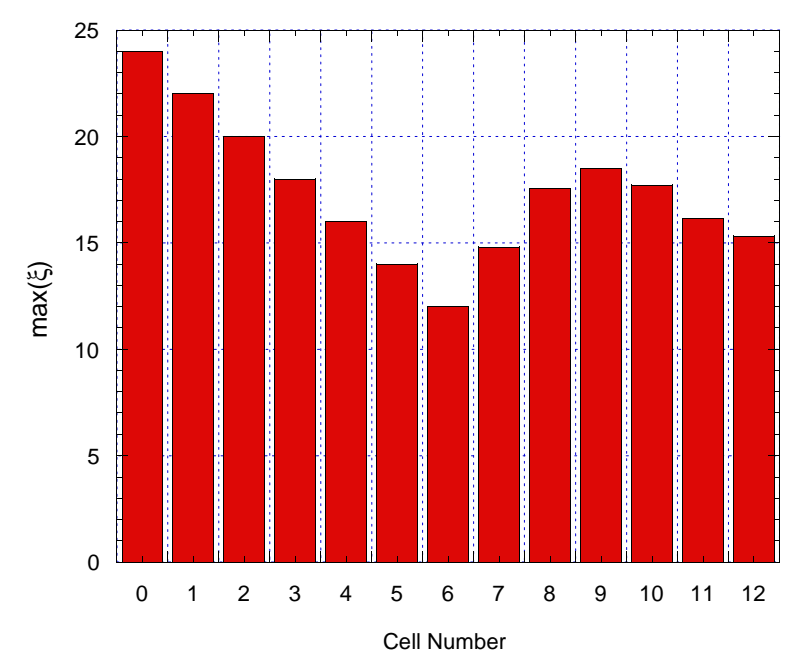

FIG. 2. (Color) The maximum spin perturbation enhancement factor is plotted against the location of a full type- 3 snake in the arc consisting of 24 FODO cells.
$0.4 \pi$ to ensure the snake's control over the spin motion [9]. Note that when the spin perturbation angle is equal to $0.5 \pi$, the initially vertical spin would rotate into the horizontal plane and regular snakes with horizontal axes would no longer be able to restore the vertical beam polarization. Thus, to ensure the spin stability in a ring one can use a pair of regular Siberian snakes plus a set of type-3 snakes to decrease the spin perturbation between the regular snakes to the required level.

\section{REGULAR SNAKE VS A TYPE-3 SNAKE}

To reduce the spin perturbation between two snakes one can also use additional regular snakes. It is quite interesting to compare the performance of a type- 3 snake with a regular Siberian snake. The spin-orbit coupling integral over an arc with a regular Siberian snake at the center can be written as

$$
\begin{aligned}
I^{ \pm}= & \int_{\frac{N}{2} \text { cells }} g \sqrt{\beta_{z}} e^{i\left(\psi \pm \psi_{z}\right)} d s \\
& +e^{i\left[\pi N\left(\nu_{1} \pm \mu_{z}\right)-2 \phi_{s}\right]} \int_{\frac{N}{2} \text { cells }} g \sqrt{\beta_{z}} e^{i\left(-\psi \pm \psi_{z}\right)} d s,
\end{aligned}
$$

where $\phi_{s}$ is the angle of the snake's axis. Note that the snake reverses both the spin direction and the direction of the spin precession; this makes the spin perturbations in a cell before and after the snake unequal. The spin-orbit integral can be rewritten as

$$
\begin{aligned}
I^{ \pm}= & \frac{1-e^{i N \pi\left(\nu_{1} \pm \mu_{z}\right)}}{1-e^{i 2 \pi\left(\nu_{1} \pm \mu_{z}\right)}} I_{\text {cell }}^{ \pm} \\
& +e^{i\left[\pi N\left(\nu_{1} \pm \mu_{z}\right)-2 \phi_{s}\right]} \frac{1-e^{i N \pi\left(-\nu_{1} \pm \mu_{z}\right)}}{1-e^{i 2 \pi\left(-\nu_{1} \pm \mu_{z}\right)}} \tilde{I}_{\mathrm{cell}}^{ \pm} .
\end{aligned}
$$

To determine the depolarization enhancement factor, we express the spin perturbation in a cell after the snake $\tilde{I}_{\text {cell }}^{ \pm}$ in terms of the spin perturbation in a cell with a "normal" spin precession direction $I_{\text {cell }}^{ \pm}$

$$
\begin{aligned}
\tilde{I}_{\mathrm{cell}}^{ \pm} & =g_{F} l \sqrt{\beta_{F}}+g_{D} l \sqrt{\beta_{D}} e^{i \pi\left(-\nu_{1} \pm \mu_{z}\right)} \\
& =I_{\mathrm{cell}}^{ \pm}+g_{D} l \sqrt{\beta_{D}}\left(e^{i \pi\left(-\nu_{1} \pm \mu_{z}\right)}-e^{i \pi\left(\nu_{1} \pm \mu_{z}\right)}\right) \\
& =I_{\mathrm{cel} 11}^{ \pm}\left(1+\frac{e^{i \pi\left(-\nu_{1} \pm \mu_{z}\right)}-e^{i \pi\left(\nu_{1} \pm \mu_{z}\right)}}{\frac{g_{F} l \sqrt{\beta_{F}}}{g_{D} l \sqrt{\beta_{D}}}+e^{i \pi\left(\nu_{1} \pm \mu_{z}\right)}}\right)
\end{aligned}
$$

where $g_{F, D}$ and $\beta_{F, D}$ are the strengths and values of the vertical beta function in the focusing and defocusing quadrupoles. Assuming focusing and defocusing quadrupoles in a cell being identical and $\sqrt{\beta_{F} / \beta_{D}} \simeq 3$, the depolarization enhancement factor can be written as 


$$
\xi=\frac{1-e^{i N \pi\left(\nu_{1} \pm \mu_{z}\right)}}{1-e^{i 2 \pi\left(\nu_{1} \pm \mu_{z}\right)}}+e^{i\left[\pi N\left(\nu_{1} \pm \mu_{z}\right)-2 \phi_{s}\right]} \frac{1-e^{i N \pi\left(-\nu_{1} \pm \mu_{z}\right)}}{1-e^{i 2 \pi\left(-\nu_{1} \pm \mu_{z}\right)}} \frac{3+e^{i \pi\left(-\nu_{1} \pm \mu_{z}\right)}}{3+e^{i \pi\left(\nu_{1} \pm \mu_{z}\right)}}
$$

With the betatron phase advance per cell being known, one can calculate the depolarization enhancement factors for each type of spin perturbation $\left(\nu_{1}+\mu_{z}\right.$ and $\nu_{1}-$ $\mu_{z}$ ). Figure 3 compares the depolarization enhancement factors for a type-3 snake and for a regular snake with the longitudinal axis of spin rotation in the region of $0.2<\nu_{1}+\mu_{z}<2$. The main spin-orbit resonances are reduced by a factor of 2 in both cases; however, the regular snake configuration is sensitive to spin perturbations when $\nu_{1} \pm \mu_{z}$ is equal to a half integer. These so-called "snake resonances" [8] do not exist with the type-3 snake configuration; therefore, type-3 snakes could have an advantage over regular Siberian snakes. Finally, note that in the presence of a regular snake, the strengths of the spin perturbation near $\nu_{1} \pm \mu_{z}=0.5$ and $\nu_{1} \pm \mu_{z}=1.5$ are different due to a weak interference effect between two types of FODO cells $\left(I_{\text {cell }}^{ \pm}\right.$and $\left.\tilde{I}_{\text {cell }}^{ \pm}\right)$. This implies that the effect of particular snake resonances could be enhanced.

So far, we have considered only the first-order spin perturbation effects. The high-order spin perturbations, which could accumulate over many turns, can apply an additional constraint on the regular snake's configuration in a ring. The effect of high-order spin perturbations is minimized when for each pair of snakes their axes are orthogonal and the spin tune is set near a half integer [9]. In this case, not only the overall spin tune is a half integer, but the spin phase advance per superperiod containing a pair of snakes is also a half integer. However, in a ring with fourfold or eightfold symmetry, two or four snake pairs with orthogonal spin rotation axes would cause an

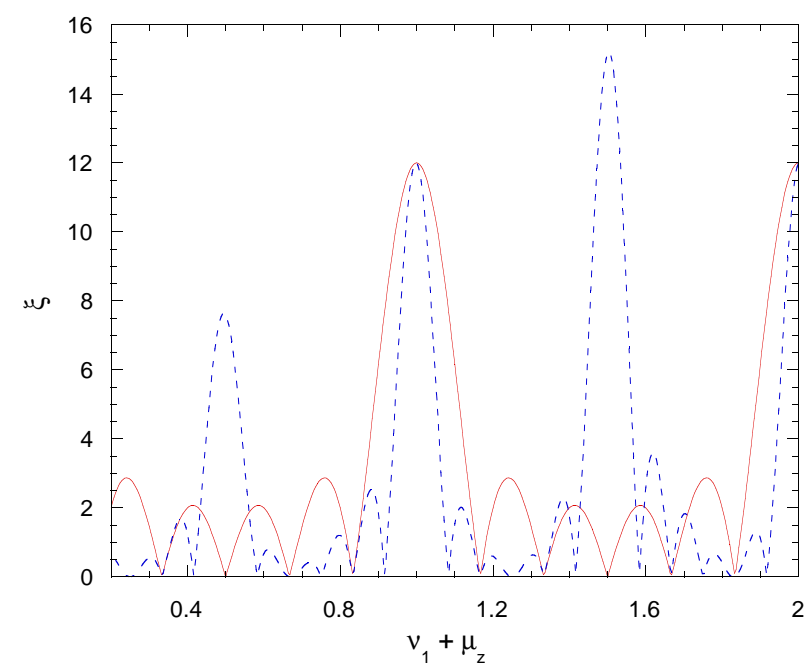

FIG. 3. (Color) The spin perturbation enhancement factor in the arc is plotted against $\nu_{1}+\mu_{z}$. The solid curve corresponds to a regular snake with the longitudinal axis at the center of the arc; the dashed curve corresponds to a full type- 3 snake installed after six cells. The arc consists of 24 FODO cells with each cell providing a $90^{\circ}$ betatron phase advance. integer spin tune, where lattice imperfections could cause beam depolarization. Since the optimal regular snake's configuration in such a ring is not applicable, installing several type-3 snakes together with a pair of regular snakes could provide better spin stability.

\section{PRACTICAL TYPE-3 SNAKES}

A practical Siberian snake for a high energy accelerator usually consists of constant field horizontal and vertical bending magnets which provide energy independent spin rotation. Any symmetric configuration of vertical and horizontal bends that restores particle orbit outside the snake would always produce a spin rotation with an axis orthogonal to the beam direction [10]. With an appropriate strength of each dipole, such a symmetric configuration could serve as a snake with either a radial or a vertical axis of spin rotation. A simple example of a symmetric type-3 snake consists of eight identical horizontally $(H)$ and vertically $(V)$ bending dipoles arranged in the following order:

$$
\hat{S}=(H,-V,-H, V, V,-H,-V, H) .
$$

A full type- 3 snake is achieved with a $90^{\circ}$ spin rotation in each magnet; the total field integral for the snake would be $21.94 \mathrm{Tm}$.

Since free space is scarce in the arcs of a high energy ring, one should also consider using helical dipoles for a compact type-3 snake design. A symmetric field configuration would require at least two helical dipoles with opposite helicity. An example of a compact helical type-3 snake is shown in Fig. 4. It consists of two helices with the same wavelength $\lambda_{h}$ separated by a horizontal dipole field insert with a length $\lambda_{h} / \pi$ and surrounded by

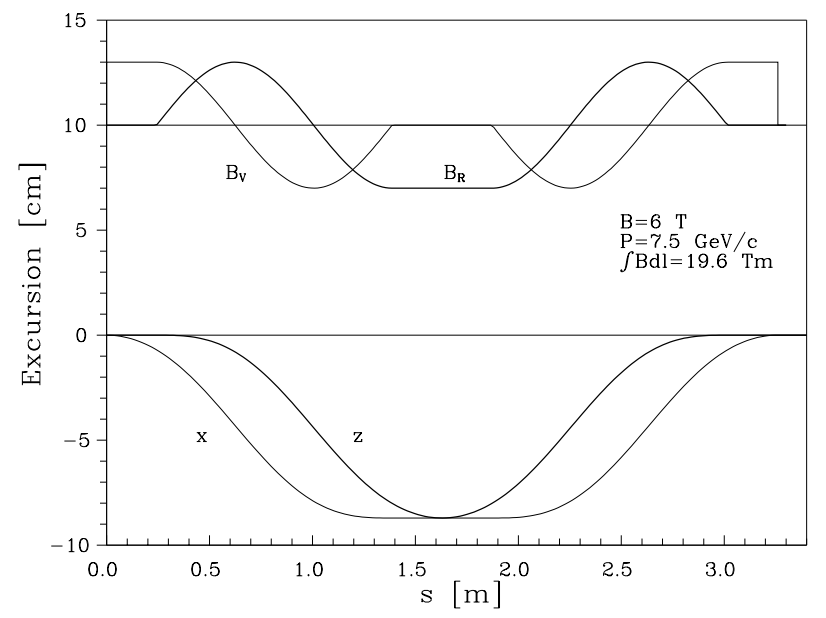

FIG. 4. Magnetic field profile and the $7.5 \mathrm{GeV} / c$ orbit excursions in the helical type- 3 snake. For $6 \mathrm{~T}$ field snake magnets, the maximum orbit excursions are $8.7 \mathrm{~cm}$ in both the vertical and the horizontal planes. 
two vertical dipole field inserts each of a $\lambda_{h} / 2 \pi$ length. With a $3 \pi / 2$ twist of the field direction in each helical magnet, this symmetric snake configuration is optically transparent for the beam motion. A full snake with $180^{\circ}$ spin rotation is obtained when, for a given field strength $B_{s}$ in the snake's magnets, the wavelength in the helical dipoles is set according to

$$
\lambda_{h}[\mathrm{~m}]=1.456 \frac{2 \pi}{B_{s}[\mathrm{~T}]} .
$$

The total field integral for the snake would be $19.55 \mathrm{~T} \mathrm{~m}$. With $6 \mathrm{~T}$ field magnets, the total snake length would be $3.26 \mathrm{~m}$. Note that the snake's axis of spin rotation is not exactly vertical but tilted by $4.57^{\circ}$ towards the radial direction; therefore, the whole snake should also be tilted by the same angle to set the vertical axis of the spin rotation.

Finally, we note that since the magnetic field direction is a continuous function of the longitudinal coordinate, the helical type- 3 snake could be manufactured either as a space saving single magnet or as a set of separate magnets.

\section{CONCLUSION}

In summary, type-3 snakes could provide an efficient way of reducing the spin perturbation near the spin depolarizing resonances. The best results could be achieved with a full type- 3 snake installed after $N / 4$ cells, where $N$ is the total number of FODO cells in the arc. Installing type-3 snakes between regular Siberian snakes in a high energy ring could be equally or, in some cases, more efficient than adding the same number of regular snakes. Thus, type- 3 snakes could be used along with the regular snakes for improving the spin stability in high energy rings such as $250 \mathrm{GeV}$ RHIC [3] at Brookhaven,
$820 \mathrm{GeV}$ HERA [11] at DESY, and $900 \mathrm{GeV}$ Tevatron [12] at Fermilab.

\section{ACKNOWLEDGMENT}

This work was supported by research grants from DESY and the U.S. Department of Energy.

[1] Ya. S. Derbenev and A. M. Kondratenko, Sov. Phys. Dokl. 20, 562 (1978); Ya. S. Derbenev et al., Part. Accel. 8, 115 (1978).

[2] A. D. Krisch et al., Phys. Rev. Lett. 63, 1137 (1989); J. E. Goodwin et al., Phys. Rev. Lett. 64, 2779 (1990).

[3] Y. Makdisi, in High Energy Spin Physics: Eleventh International Symposium, edited by K. Heller and S. Smith, AIP Conf. Proc. No. 343 (AIP, New York, 1995), p. 75.

[4] R. Pollock, Nucl. Instrum. Methods Phys. Res., Sect. A 300, 210 (1991).

[5] A. W. Chao and K. Yokoya, KEK Report No. KEK 81-7, 1981.

[6] K. Steffen, Part. Accel. 24, 48 (1988).

[7] A. W. Chao and Ya.S. Derbenev, Part. Accel. 36, 25 (1991).

[8] S. Y. Lee and S. Tepikian, Phys. Rev. Lett. 56, 1635 (1986); R. A. Phelps et al., Phys. Rev. Lett. 78, 2772 (1997).

[9] S. Y. Lee and E. D. Courant, Phys. Rev. D 41, 292 (1990).

[10] Yu. M. Ado and V.A. Anferov, Sov. Phys. Usp. 37, 1147 (1994).

[11] SPIN Collaboration and DESY Polarization Team, University of Michigan Report No. UM-HE 96-20, 1996.

[12] SPIN Collaboration, University of Michigan Report No. UM-HE 95-09, 1995. 